\section{Food allergens: state of the art in Piedmont region in the period 2011-2012}

\author{
Antonio Barbaro, ${ }^{1}$ Francesca Rubinetti, ${ }^{1}$ \\ Annamaria Galleggiante Crisafulli, ${ }^{1}$ \\ Maria Cristina Radaelli, ${ }^{1}$ Laura Chiavacci, ${ }^{1}$ \\ Daniela Manila Bianchi, ${ }^{2}$ \\ Daniela Adriano, ${ }^{2}$ Fabio Zuccon, ${ }^{2}$ \\ Sandra Fragassi, 2 \\ Giuseppina Buonincontro, ${ }^{2}$ \\ Walter Vencia, ${ }^{2}$ Lucia Decastelli² \\ 'Struttura Complessa Epidemiologia \\ e Osservatorio Epidemiologico, \\ Istituto Zooprofilattico Sperimentale \\ del Piemonte, Liguria e Valle d'Aosta; \\ 2Struttura Complessa Controllo Alimenti \\ e Igiene delle Produzioni, Istituto \\ Zooprofilattico Sperimentale del \\ Piemonte, Liguria e Valle d'Aosta, Torino, \\ Italy
}

\section{Abstract}

The US National Institutes of Allergy and Infectious Diseases defines food allergy as adverse health effect arising from a specific immune response that occurs reproducibly on exposure to a given food. Undeclared allergens in food label represent a risk for consumers, as there is no therapy for food allergies. According to Directive 2003/89/EC, declaration of all ingredients and derived substances in the label is mandatory. In 2011-2012, in Piedmont region (North-western Italy) 285 food samples were analysed for $\beta$-lactoglobulin and 234 for egg proteins. The aim of this work was to analyse 2 years data in order to assess the presence of undeclared milk and egg allergenic proteins in food placed on the market checking the compliance of labeling of food allergens. Analyses were carried out with ELISA tests, both for the detection of the egg and milk proteins. $\beta$-lactoglobulin was found in $2.8 \%(8 / 286)$ of samples, while egg proteins in $4.7 \%(11 / 234)$

\section{Introduction}

The US National Institutes of Allergy and Infectious Diseases (NIAID) defines food allergy as adverse health effect arising from a specific immune response that occurs reproducibly on exposure to a given food (Boyce et al., 2010). Undeclared allergens in food label represent a risk for consumers, as there is no therapy for food allergies. According to Directive
2003/89/EC (European Commission, 2003) declaration of all ingredients and derived substances in the food label is mandatory.

A systematic review concerning the epidemiology of food allergies has concluded that in the United States food allergies affect more than $1-2 \%$ but less than $10 \%$ of the population. The number of cases of food allergy varies by age, geographic area, diet and other factors that make difficult a reliable evaluation of the prevalence of food allergies (Sicherer, 2011). Even in Europe, percentages do not change from those found in the United States (Rona et al., 2007).

The most important foods responsible for allergies are: milk, eggs, peanuts, shellfish, fish, soy, nuts and cereals; allergies to additives are less common (Sicherer and Sampson, 2010; Young et al., 1994). The only way to protect allergic subject health is to avoid the ingestion of allergenic substances, therefore the label, which shows the presence of all ingredients and allergens, is an essential tool of food safety.

The Directive 2003/89/EC (European Commission, 2003), implemented in Italy by Legislative Decree 114/2006 (Italian Republic, 2006, 2006), provides information to allergic consumers, making a list of allergens that must be indicated on the label, and requires the declaration of all substances derived from the main allergens. Moreover, the Directive takes into account the case of a possible unintentional presence of allergenic substances in products and allows to the food business operators the use of phrases such as it may contain traces of ... or produced in a factory where XY are in use ... The substances included in the list of Directive 2003/89/EC are: cereals containing gluten, crustaceans, eggs and egg products, fish, peanuts, soy, milk, nuts, celery, mustard, sesame seeds, sulfur dioxide and sulphites at concentrations above $10 \mathrm{mg} / \mathrm{kg}$ or $10 \mathrm{mg} / \mathrm{L}$ expressed as $\mathrm{SO}_{2}$. The list was supplemented by Directive 2006/142/EC (European Commission, 2003), which added the obligation to indicate also lupine, molluscs and products thereof.

Since 2007, in Piedmont region (Northwestern Italy) a monitoring plan for the detection of allergens in food was issued in order to monitor the presence of any non-compliance on the labels. This paper presents the results of the monitoring plans carried out in the period 2011-2012.

\section{Materials and Methods}

In 2011-2012 meat products, meat preparations and minced meat, sweet and salty snacks, baby food, ready-to-eat (RTE) products and other foodstuffs were tested for egg proteins and $\beta$-lactoglobulin. Samples were collec-
Correspondence: Antonio Barbaro, Struttura Complessa Epidemiologia e Osservatorio Epidemiologico, Istituto Zooprofilattico Sperimentale del Piemonte, Liguria e Valle d'Aosta, via Bologna 148, 10154 Torino, Italy. Tel: +39.011.2686346 - Fax: +39.011 .2485831$

E-mail: antonio.barbaro@izsto.it

Key words: Food allergens, Egg proteins, Milk proteins.

Received for publication: 12 May 2013. Accepted for publication: 1 November 2013

This work is licensed under a Creative Commons Attribution 3.0 License (by-nc 3.0)

(C) Copyright A. Barbaro et al., 2014 Licensee PAGEPress, Italy

Italian Journal of Food Safety 2014; 3:1700

doi:10.4081/ijfs.2014.1700

ted by the Veterinary Services and Food Hygiene and Nutrition Services at food retails of Piedmont region and they were selected based on the absence of information about the presence of allergens in the labels. The sampling was performed according to the procedures of the official sampling plans, providing four or five aliquots.

The analyses were performed at the Food Control Laboratory of the Veterinary Epidemiology Centre of Piedmont, Liguria and Valle d'Aosta (Istituto Zooprofilattico del Piemonte, Liguria e Valle d'Aosta), Turin, Italy. The methods used are shown in Table 1. Both kits are based on similar analytical procedures. Briefly, $1 \mathrm{~g}$ of sample and $20 \mathrm{~mL}$ of extraction buffer, specific for the allergen target and provided with the kit, were homogenised by Stomaker®. Therefore, an incubation in a water bath at $60^{\circ} \mathrm{C}$ for 10 min was set up and, later, sample was filtrated or centrifuged. An aliquot of $100 \mu \mathrm{L}$ of the supernatant was added into the wells of a plate, in which specific antibodies were immobilised. After careful washing, the secondary antibody, conjugated with peroxidase, was added. The complex antibody/antigen/antibody (sandwich) was detected by the addition of the chromogenic substrate that determines a colorimetric reaction. The reading of this reaction was performed using a spectrophotometer at $450 \mathrm{~nm}$ of absorbance and samples concentration was calculated according to the Lambert-Beer law with the aid of the specific programme RIDAWIN ${ }^{\circledR}$ Software. The detection limit for the ELISA kit RIDASCREEN ${ }^{\circledR}$ FAST $\beta$-lactoglobulin is 0.5 $\mathrm{mg} / \mathrm{kg}$ and for the ELISA kit RIDASCREEN ${ }^{\circledR}$ FAST Ei/ Egg Protein is $0.6 \mathrm{mg} / \mathrm{kg}$. 


\section{Results}

In the period 2011-2012, 285 food samples for $\beta$-lactoglobulin and 234 for egg protein were tested (Tables 2 and 3). Overall, 19 samples exceeded the limit of detection and were classified as non-compliant: in particular, 8 out of $285(2.8 \%)$ for $\beta$-lactoglobulin and 11 out of 234 (4.7\%) for egg protein.

The non-compliance for $\beta$-lactoglobulin covered the $5.1 \%(4 / 78)$ of meat preparations and minced meat, $1.7 \%(2 / 117)$ of meat products, $2.5 \%(1 / 40)$ of sweet and salty snacks, and $4.3 \%$ (1/23) of baby food. Traces of egg protein were detected in $8.9 \%$ (7/78) of meat preparations and minced meat, in 1.9\% (2/107) of meat products and $8.3 \%(2 / 24)$ of sweet and salty snacks.

\section{Discussion}

Some allergens play a critical technological role in food industry and their presence in food can result especially from accidental contamination, as well as voluntary use as an ingredient. In some plants it may be difficult to separate the production lines including the use of allergenic ingredients from those that must be free. The presence of $\beta$-lactoglobulin and egg protein, especially in meat preparations and minced meat, could be probably attributed to an involuntary contamination. For that reason, the food business operators should increase the level of attention with careful application of selfmonitoring plan and implement training programmes of staff in order to ensure the health of allergic consumers.

Table 1. Methods used to detect food allergens.

\begin{tabular}{lccc} 
Allergen & Method & Manufacturer & LOD (ppm) \\
$\beta$-lactoglobulin & ELISA & r-biopharm (Darmstadt, Germany) & 0.5 \\
Egg protein & ELISA & r-biopharm & 0.6 \\
\hline
\end{tabular}

LOD, limit of detection.

Table 2. Sample types tested for $\beta$-lactoglobulin.

\begin{tabular}{lccc} 
Samples type & Total samples (n) & Non-compliant samples \\
Meat products & & n & $\%$ \\
Meat preparation and minced meat & 117 & 2 & 1.7 \\
\hline Snack food & 78 & 4 & 5.1 \\
Baby food & 40 & 1 & 2.5 \\
\hline RTE products & 23 & 1 & 4.3 \\
Other foodstuff & 17 & 0 & 0 \\
Total & 10 & 0 & 0 \\
\hline
\end{tabular}

RTE, ready-to-eat.

Table 3. Sample types tested for egg proteins.

\begin{tabular}{lccc} 
Samples type & Total samples (n) & Non-compliant samples \\
& & n & $\%$ \\
Meat products & 107 & 2 & 1.9 \\
Meat preparation and minced meat & 78 & 7 & 8.9 \\
\hline Snack food & 24 & 2 & 8.3 \\
Baby food & 4 & 0 & 0 \\
\hline RTE products & 14 & 0 & 0 \\
Other foodstuff & 7 & 0 & 0 \\
\hline Total & 234 & 11 & 4.7 \\
\hline RTE, ready-to-eat. & & &
\end{tabular}

RTE, ready-to-eat.

Rona RJ, Keil T, Summers C, Gislason D, Zuidmeer L, Sodergren E, Sigurdardottir ST, Lindner T, Goldhahn K, Dahlstrom J, McBride D, Madsen C, 2007. The prevalence of food allergy: a meta-analysis. J Allergy Clin Immun 120:638-46.

Sicherer SH, 2011. Epidemiology of food allergy. J Allergy Clin Immun 127:594-602.

Sicherer SH, Sampson HA, 2010. Food allergy. J Allergy Clin Immun 125(Suppl.2):116-25.

Young E, Stoneham MD, Petruckevitch A, Barton J, Rona R, 1994. A population study of food intolerance. Lancet 343:1127-30. 